\title{
A review of charm semileptonic decays
}

\author{
Doris Yangsoo Kim ${ }^{\mathrm{a}}$ \\ ${ }^{a}$ University of Illinois at Urbana-Champaign, 1110 W Green St., Urbana, IL. 61801, US
}

Several high energy experiments have been actively pursuing the analyses of semileptonic decays of charm mesons, resulting in numerous new results and publications. In this report, we summarize the recent efforts on the topics of pseudoscalar and vector charm semileptonic decays, especially in the area of branching fractions and form factor measurements.

\section{INTRODUCTION}

The semileptonic decays of charm particles continue to be an important area of research. The branching fractions provide measurements of CKM matrix elements in the charm sector, $\left|V_{c q}\right|^{2}$. All hadronic complications are contained in the form factors, which can be calculated via nonperturbative lattice QCD, Heavy Quark Effective Theories, Quark Models, and other methods. By comparing experimental observations with the the lattice QCD calculations, we obtain a high quality lattice calibration, which is crucial in reducing systematic uncertainties in the unitarity triangle. The same technique validated by charm decays can be applied to beauty decays, subsequently improving the CKM matrix elements in the beauty sector.

Several experiments study charm particle decays, including charm and $B$-factories and fixed target experiments. The CLEO-c experiment started taking data recently and has collected $281 \mathrm{pb}^{-1}$ at $\Psi(3770)$. Since this energy is the threshold for the $D^{+} D^{-}$and $D^{0} \bar{D}^{0}$ production, the number of decay particles in the detector is relatively small, the event reconstruction environment is quite clean, particle identification is excellent, and backgrounds are negligible. Last year, they reported branching fraction measurements of semileptonic charm decays using the first 56 $\mathrm{pb}^{-1}$ sample [1]. Their results are already comparable or better than the PDG 2004 world averages [2].

\section{INCLUSIVE SEMILEPTONIC BRANCHING FRACTIONS}

CLEO-c (06) 3] reported both new measurements on the inclusive branching fractions of $D^{0} \rightarrow X e^{+} \nu_{e}$ and $D^{+} \rightarrow X e^{+} \nu_{e}$ decays and the corresponding electron momentum spectra based on the $281 \mathrm{pb}^{-1}$ sample collected at the $\Psi(3770)$. At least $200 \mathrm{MeV} / c$ is required for the electron momentum. The $D^{0}$ and $D^{+}$spectra look similar to each other, as expected by theories. The spectra are extrapolated for the lower momentum region to obtain the branching fraction numbers. The results are: $(6.46 \pm 0.17 \pm 0.13) \%$ and $(16.13 \pm 0.20 \pm 0.33) \%$ for the branching fractions of the $D^{0} \rightarrow X e^{+} \nu_{e}$ and $D^{+} \rightarrow X e^{+} \nu_{e}$ decays, respectively. The sums of the known exclusive semielectronic decay modes are $(6.1 \pm 0.2$ $\pm 0.2) \%$ for $D^{0}$ and $(15.1 \pm 0.5 \pm 0.5) \%$ for $D^{+}$. Hence, the sums of the exclusive branching fractions come close to saturating the inclusive branching fractions, leaving little room for any undiscovered semileptonic decays.

\section{A FADING ENIGMA ON THE RATIO OF VECTOR TO PSEUDOSCALAR DECAY RATES}

There has been a disagreement on the decay width ratio of the vector semileptonic decays relative to the pseudoscalar semileptonic decays: $\Gamma\left(D \rightarrow K^{*} \ell \nu_{\ell}\right) / \Gamma\left(D \rightarrow K \ell \nu_{\ell}\right)$. The earlier theoretical predictions for this ratio from late 80 's and early 90 's were found to be between 0.5 to 1.1, which are 50 to $100 \%$ larger than the experimental measurements at that time. This difference 
between the theory and the data was attributed to difficulties in computing the axial-vector form factor $\left(A_{1}\right)$. Since mid 90's, the updated branching ratio predictions are between 0.5 to 0.7 , being closer to the experimental results.

Recently, several experiments revisited this vector-pseudoscalar anomaly problem. FOCUS (04) 4] and CLEO (05) [1] reported this ratio as $0.594 \pm 0.05$ and $0.63 \pm 0.04$, respectively. This year BES [56] reported two measurements: $0.57 \pm 0.17$ for the $D^{+}$channel and $0.74 \pm 0.40$ (preliminary) for the $D^{0}$ channel. These new sets of measurements are consistent with one another and with the updated predictions. This old anomaly appears to be fading away.

\section{FORM FACTORS OF PSEU- DOSCALAR DECAYS}

The differential decay rate for a $D$ meson decaying into a pseudoscalar meson, a lepton and a neutrino is given by Eq. [1]

$\frac{d \Gamma\left(D \rightarrow P \ell \nu_{\ell}\right)}{d q^{2}}=\frac{G_{F}^{2}\left|V_{c q}\right|^{2} P_{P}^{3}}{24 \pi^{3}}\left|f_{+}\left(q^{2}\right)\right|^{2}+O\left(m_{\ell}^{2}\right)$,

where $q^{2}$ is the invariant mass of the leptonneutrino pair. The pseudoscalar semileptonic process should provide both a clean measurement of CKM angles and a powerful test of lattice QCD. Unfortunately, the QCD test is compromised somewhat since the decay rate vanishes at the highest $q^{2}$ where the sensitivity to the form of $f_{+}\left(q^{2}\right)$ is greatest. The highest $q^{2}$ area is also the zero recoil limit region for the quark inside the meson where theory calculations can be obtained relatively easy.

\subsection{Parameterization of form factor mea- surements}

To handle the measured $q^{2}$ distributions properly, several fit parameterization have been suggested. Based on the dispersion relation, the form factor $f_{+}\left(q^{2}\right)$ can be described by a pole term and an integral such as,

$f_{+}\left(q^{2}\right)=\frac{\mathcal{R}}{m_{D^{*}}^{2}-q^{2}}+\frac{1}{\pi} \int_{\left(m_{D}+m_{P}\right)^{2}}^{\infty} \frac{\operatorname{Im} f_{+}(s)}{s-q^{2}-i \varepsilon} d s(2)$

where $m_{D}^{*}, m_{D}$ and $m_{P}$ are the masses of the excited spin-1 $D$ meson, the decaying $D$ meson, and the pseudoscalar meson, respectively. In the past, only a "single" pole term was used to fit the data distribution. But the world average of the fitted pole mass for the $D^{0} \rightarrow K^{-} \ell^{+} \nu_{\ell}$ decay turned out to be $5.1 \sigma$ lower than the expected value, $m_{D_{S}^{*}}$, underscoring the importance of the integral term.

Becirevic and Kaidalov [7] represented the integral by an additional, effective pole which leads to a parameterization of $f_{+}\left(q^{2}\right)=f_{+}(0) /\{(1-$ $\left.\left.q^{2} / m_{D^{*}}^{2}\right)\left(1-\alpha q^{2} / m_{D^{*}}^{2}\right)\right\}$. This scheme is called the modified pole form. Recently, Hill 8 proposed a less model dependent way of dealing with the $f_{+}$analytic singularities. He makes a complex mapping from $q^{2}$ to $z$, which pushes the cut singularities far from the physical $q^{2}$ region. In the $z$ space, the $f_{+}(z)$ distribution looks linear and can be represented by a rapidly converging Taylor series. He applied the method to the recent beauty and charm data sets. The results are given in Reference [8].

\subsection{Experimental results from BABAR, BELLE, CLEO, and FOCUS}

The current generation of experiments have produced data sets. Not only are parametric fits made to various $q^{2}$ forms, often the $q^{2}$ distributions are presented non-parametrically, making the visible comparison to the most recent lattice QCD results (05) possible 9]. The $\alpha$ measurements from present experiments are summarized in Table 11

FOCUS (05) measured the form factors of the $D^{0} \rightarrow K^{-} \mu^{+} \nu_{\mu}$ and $D^{0} \rightarrow \pi^{-} \mu^{+} \nu_{\mu}$ decays and presented the non-parametric $f_{+}\left(q^{2}\right)$ distribution of the $D^{0} \rightarrow K^{-} \mu^{+} \nu_{\mu}$ decay based on a sample of 13,000 events [10. After subtracting known charm backgrounds, their $f_{+}\left(q^{2}\right)$ distribution is an excellent match to a pole form with $m_{\text {pole }}=$ $1.91 \pm 0.04 \pm 0.05 \mathrm{GeV} / c^{2}$ or to a modified pole form with $\alpha=0.32$.

BELLE (06) measured the absolute branching fractions and form factors of the $D^{0} \rightarrow K^{-} \ell^{+} \nu_{\ell}$ and $D^{0} \rightarrow \pi^{-} \ell^{+} \nu_{\ell}$ decays using both muonic and electronic modes [11. By fully reconstructing the recoil charm meson and the other mesons from fragmentation, they ensured an excellent $q^{2}$ resolution and a low level of backgrounds al- 
Table 1

Summary of recent experimental results on the form factor studies of $D \rightarrow K \ell \nu_{\ell}$. Only the fit parameter $\alpha$ of the modified pole form is shown here. CLEO-c obtained a low $\alpha$ while BABAR and BELLE obtained high numbers compared with other experiments. For other fit results and information on $D \rightarrow \pi \ell \nu_{\ell}$ decays, check the reference for each experiment.

\begin{tabular}{l|l|l}
\hline Experiment & $\alpha$ & Channel \\
\hline CLEO III [14] & $0.36 \pm 0.10_{-0.07}^{+0.08}$ & $D^{0}$ to $e, \mu$ \\
FOCUS [10] & $0.28 \pm 0.08 \pm 0.07$ & $D^{0}$ to $\mu$ \\
BELLE 11] & $0.40 \pm 0.12 \pm 0.09$ & $D^{0}$ to $e$ \\
& $0.66 \pm 0.11 \pm 0.09$ & $D^{0}$ to $\mu$ \\
BABAR 12] & $0.43 \pm 0.03 \pm 0.04$ & $D^{0}$ to $e$ \\
CLEO-c [13] & $0.19 \pm 0.05 \pm 0.03$ & $D^{0}$ to $e$ \\
& $0.20 \pm 0.08 \pm 0.04$ & $D^{+}$to $e$ \\
\hline
\end{tabular}

beit with considerably reduced statistics. They obtained 2,700 $D^{0} \rightarrow K^{-} \ell^{+} \nu_{\ell}$ and $300 D^{0} \rightarrow$ $\pi^{-} \ell^{+} \nu_{\ell}$ events from a $282 \mathrm{fb}^{-1}$ data sample. Their data set was fit to the simple pole form and the modified pole form.

BABAR (06) reported a preliminary measurement of the form factors of the $D^{0} \rightarrow K^{-} e^{+} \nu_{e}$ decay [12. By applying the $D^{*}$ tag, they obtained a sample of 100,000 events based on a 75 $\mathrm{fb}^{-1}$ data sample. They also used the simple pole form and the modified pole form to fit their data.

CLEO (06) reported preliminary measurements of the absolute branching fractions and form factors of $D^{0} \rightarrow K^{-} e^{+} \nu_{e}, D^{0} \rightarrow \pi^{-} e^{+} \nu_{e}$, $D^{+} \rightarrow K^{0} e^{+} \nu_{e}$, and $D^{+} \rightarrow \pi^{0} e^{+} \nu_{e}$ decays based on a sample of $281 \mathrm{pb}^{-1}$ [13]. They did not require the recoil side $\bar{D}$ tagging, which enhanced the size of the selected events with a slight penalty in the $q^{2}$ resolution. Both the simple and modified pole forms are used for fitting.

\section{FORM FACTORS OF VECTOR DE- CAYS}

The kinematics of $D \rightarrow \bar{K}^{*} \ell \nu_{\ell}$ decays is determined by form factors and five kinematic variables: $q^{2}, m(K \pi)$, the lepton decay angle $\theta_{\ell}$, the vector decay angle $\theta_{V}$, and the acoplanarity between two decay planes $\chi$. For this type of de- cays, Körner and Schuler [15] proposed a set of helicity-basis form factors $H_{+}\left(q^{2}\right), H_{-}\left(q^{2}\right)$, and $H_{0}\left(q^{2}\right)$, which are, in principle, computable by QCD theories. After integrating over $\chi$, the decay intensity of the $D^{+} \rightarrow K^{-} \pi^{+} \ell^{+} \nu_{\ell}$ process becomes proportional to

$$
q^{2}\left[\begin{array}{l}
\left(\left(1+\cos \theta_{\ell}\right) \sin \theta_{V}\right)^{2}\left|H_{+}\left(q^{2}\right)\right|^{2}|B W|^{2} \\
+\left(\left(1-\cos \theta_{\ell}\right) \sin \theta_{V}\right)^{2}\left|H_{-}\left(q^{2}\right)\right|^{2}|B W|^{2} \\
+\left(2 \sin \theta_{\ell} \cos \theta_{V}\right)^{2}\left|H_{0}\left(q^{2}\right)\right|^{2}|B W|^{2} \\
+8\left(\sin \theta_{\ell}^{2} \cos \theta_{V}\right) H_{0}\left(q^{2}\right) h_{0}\left(q^{2}\right) R e\left\{A e^{-i \delta} B W\right\} \\
+O\left(A^{2}\right)+m_{\ell} \text { terms }
\end{array}\right]
$$

where $B W$ and $A e^{-i \delta}$ represent the $\bar{K}^{*}$ resonance and the $s$-wave in the $K-\pi$ system, respectively. A new form factor $h_{0}\left(q^{2}\right)$ for the $s$-wave is introduced to describe the interference between the $s$-wave and $\bar{K}^{*}$. In the Körner and Schuler scheme, $H_{ \pm}$and $H_{0}$ are linear combinations of two axial-vector and one vector form factors. In the past, it was assumed that the axial- and vector form factors take the simple pole form such as $A_{i}\left(q^{2}\right)=A_{i}(0) /\left(1-q^{2} / M_{A}^{2}\right)$ and $V\left(q^{2}\right)=$ $V(0) /\left(1-q^{2} / M_{V}^{2}\right)$. With $M_{V}$ and $M_{A_{i}}$ fixed at 2.1 and $2.5 \mathrm{GeV} / c^{2}$, experiments measured the ratio $R_{V}=V(0) / A_{1}(0)$ and $R_{2}=A_{2}(0) / A_{1}(0)$ from their data sets. Recently, following the idea of Becirevic and Kaidalov [7, Fajfer and Kamenik [16] proposed a new parameterization for the vector semileptonic decays, where some of the form factors are replaced by effective poles.

The world averages of the form factor ratios of the $D^{+} \rightarrow \bar{K}^{* 0} \ell^{+} \nu_{\ell}$ decays are: $R_{V}=1.66 \pm 0.060$ and $R_{2}=0.827 \pm 0.055$ [2. The experimental results are based on the simple pole parameterization and fairly consistent among themselves with small uncertainties. However, there hasn't been any serious experimental check on the shape of the form factors yet.

Based on their large data samples, FOCUS (05) 17 and CLEO (06) 18 obtained details on the shape of the helicity form factors. The functional form of Eq. 3 shows that one can disentangle the helicity form factors based on their different angular bin populations. Monte Carlo simulations are used to calculate the projection weights as a function of decay angles, The form factors $H_{+}^{2}\left(q^{2}\right), H_{-}^{2}\left(q^{2}\right), H_{0}^{2}\left(q^{2}\right)$, and $h_{0}\left(q^{2}\right) H_{0}\left(q^{2}\right)$ are obtained from projectively weighted histograms. 
CLEO compared the shape of $H_{+}^{2}\left(q^{2}\right), H_{-}^{2}\left(q^{2}\right)$, $H_{0}^{2}\left(q^{2}\right)$, and $h_{0}\left(q^{2}\right) H_{0}\left(q^{2}\right)$ to a parametric model based on a FOCUS paper 19 and found a good agreement except the interference term. The presence of the $s$-wave interference is confirmed, but there is a small deviation from the model in the $h_{0}\left(q^{2}\right) H_{0}\left(q^{2}\right)$ shape. More data would be needed to obtain detailed information on the shape of $h_{0}\left(q^{2}\right)$. CLEO also compared their data to a model based on constant axial- and vector form factors. The data fits this model equally well, implying little sensitivity to the simple pole masses with present statistics.

\section{SUMMARY}

The field of the semileptonic charm decay is very active. Both inclusive and exclusive branching fractions are updated recently. Several experiments are involved in a healthy competition to measure charm semileptonic form factors as accurately as possible. In light of recent experimental progress, new ideas on fit parameterization ideas and lattice QCD calculations are emerging.

The topics covered in this article is just part of the story. More updates on exclusive branching fractions, rare decays, $D_{S}$ semileptonic decays and form factor measurements will appear in near future.

\section{REFERENCES}

1. CLEO Collaboration, G.S. Huang et al., Phys. Rev. Lett.95 (2005) 181801, arXiv:hep-ex/0506053; CLEO Collaboration, T.E. Coan et al., Phys. Rev. Lett.95 (2005) 181802, arXiv:hep-ex/0506052.

2. S. Eidelman et al., Phys. Lett. B592 (2004) 1.

3. CLEO Collaboration, N.E. Adam et al., arXiv:hep-ex/0604044.

4. FOCUS Collaboration, J.M. Link et al., Phys. Lett. B598 (2004) 33.

5. BES Collaboration, M. Ablikim et al., Eur. Phys. J. C47 (2006) 31, arXiv:hep-ex/0605103.

6. BES Collaboration, H.L. Ma, a talk presented at International Workshop on Tau-
Charm Physics, June 05-07, 2006, Beijing, China.

7. D. Becirevic and A.B. Kaidalov, Phys. Lett. B478 (2000) 417.

8. R. Hill, arXiv:hep-ph/0606023 and references therein.

9. C. Aubin et al., Phys. Rev. Lett.textbf94 (2005) 011601, arXiv:hep-ph/0408036; M. Okamoto et al., a talk presented at the XXII International Symposium on Lattice Field Theory, June 21-26, 2004, Fermilab, arXiv:hep-lat/0409116.

10. FOCUS Collaboration, J.M. Link et al., Phys. Lett. B607 (2005) 233, arXiv:hep-ex/0410037.

11. BELLE Collaboration, L. Widhalm et al., arXiv:hep-ex/0604049.

12. BABAR Collaboration, B. Aubert et al., arXiv:hep-ex/0607077.

13. J. Wiss, a talk presented at Flavor Physics and CP Violation Conference, April 9-12, 2006, Vancouver, Canada, arXiv:hep-ex/0605030.

14. CLEO Collaboration, G.S. Huan et al., Phys. Rev. Lett.94 (2005) 011802, arXiv:hep-ex/0407035.

15. J.G. Körner and G.A. Schuler, Z. Phys. C38 (1988) 511 [Erratum-ibid, C 41 (1989) 690].

16. S. Fajfer and J. Kamenik, Phys. Rev. D72 (2005) 034029, arXiv:hep-ph/0506051.

17. FOCUS Collaboration, J.M. Link et al., Phys. Lett. B633 (2006) 183, arXiv:hep-ex/0509027.

18. CLEO Collaboration, M.R. Shepherd et al., arXiv:hep-ex/0606010.

19. FOCUS Collaboration, J.M. Link et al., Phys. Lett. B544 (2002) 89. 